\title{
Probing the stellar population of seyfert galaxies: a near infrared perspective
}

\author{
Rogério Riffel $^{1}$, Miriani G. Pastoriza ${ }^{1}$, Alberto Rodríguez-Ardila ${ }^{2} \&$ \\ Charles Bonatto ${ }^{1}$ \\ ${ }^{1}$ Departamento de Astronomia, Universidade Federal do Rio Grande do Sul. Av. Bento \\ Gonçalves 9500, Porto Alegre, RS, Brazil. \\ email: riffel, miriani.pastoriza, charles.bonatto @ufrgs.br \\ ${ }^{2}$ Laboratório Nacional de Astrofísica/MCT - Rua dos Estados Unidos 154, Bairro das Nacões, \\ Itajubá, MG, Brazil. \\ email: aardila@lna.br
}

\begin{abstract}
We employ IRTF SpeX NIR $(0.8-2.4 \mu \mathrm{m})$ spectra to investigate the stellar population (SP), active galactic nuclei (AGN), featureless continuum $(F C)$ and hot dust properties in 9 Sy 1 and 15 Sy 2 galaxies. Both the STARLIGHT code and the hot dust as an additional base element were used for the first time in this spectral range. Our synthesis shows significant differences between Sy 1 and Sy 2 galaxies: the hot dust component is required to fit the $K$-band spectra of $\sim 90 \%$ of the Sy 1 galaxies, and only of $\sim 25 \%$ of the Sy 2; about $50 \%$ of the Sy 2 galaxies require an $F C$ component contribution $\gtrsim 20 \%$; this fraction increases to about $60 \%$ in the Sy 1 . In about $50 \%$ of the Sy2, the combined FC and young components contribute with more than $20 \%$, while this occurs in $90 \%$ of the Sy1, suggesting recent star formation in the central region. The central few hundred parsecs of our galaxy sample contain a substantial fraction of intermediate-age SPs with a mean metallicity near solar. Our SP synthesis confirms that the $1.1 \mu \mathrm{m} \mathrm{CN}$ band can be used as a tracer of intermediate-age stellar populations.
\end{abstract}

Keywords. galaxies: active, galaxies: stellar content, stars: AGB and post-AGB.

\section{Introduction}

To determine if circumnuclear stellar populations (SPs) and nuclear activity are closely related phenomena, or if they are only incidental, it is of utmost importance the correct characterisation of the former, since a substantial fraction of the energy emitted by a galaxy in the optical to near-infrared (NIR) domain is starlight. Moreover, the analysis of the stellar content provides information on critical processes such as the star formation episodes and the evolutionary history of the galaxy.

One reason to use the NIR to study the SP of AGNs is that it is the most convenient spectral region accessible to ground-based telescopes to probe highly obscured sources. However, tracking the star formation in the NIR is complicated (Origlia \& Oliva 2000). Except for a few studies such as those based on the $\mathrm{Br} \gamma$ emission or the $\mathrm{CO}(2-0)$ first overtone (e.g. Origlia et al. 1993), the SP of the inner few hundred parsecs of active galaxies in the NIR remains poorly known (Riffel et al. 2008, 2009). Because stellar absorption features in the NIR are widely believed to provide a means for recognizing red supergiants (Oliva et al. 1995), they arise as prime indicators for tracing starbursts in galaxies. Besides the short-lived red supergiants, the NIR also includes the contribution of thermally- pulsating asymptotic giant branch (TP-AGB) stars, enhanced in young to intermediate age stellar populations $(0.2 \leqslant t \leqslant 2$ Gyr, Maraston 2005). The TP-AGB phase becomes fully developed in stars with degenerate carbon oxygen cores (see Iben \& Renzini 1983, for a review). Evidence of this population in the optical is usually missed, 
as the most prominent spectral features associated with this population fall in the NIR Maraston (2005).

With the new generations of Evolutionary Population Synthesis (EPS) models, which include a proper treatment of the TP-AGB phase (Maraston 2005), it is now possible to study the NIR SP of galaxies in more detail. According to these models, the effects of TPAGB stars in the NIR spectra are unavoidable. Maraston (2005) models, by including empirical spectra of oxygen-rich stars (Lançon \& Wood 2000), are able to foresee the presence of NIR absorption features such as the 1.1 $\mu \mathrm{m} \mathrm{CN}$ band (Riffel et al. 2007), whose detection can be taken as an unambiguous evidence of a young to intermediate age stellar population.

\section{Spectral Synthesis}

Clearly, the most important ingredient in the SP synthesis is the spectral base set, $b_{j, \lambda}$. An ideal base of elements should cover the range of spectral properties observed in the galaxy sample, providing enough resolution in age and metallicity to properly address the desired scientific question (Schmidt et al. 1991,Cid Fernandes et al. 2005).

One improvement here over previous approaches that attempted to describe the stellar content of active galaxies using NIR spectroscopy is the inclusion of EPS models that take into account the effects of TP-AGB stars. Accordingly, we use as base set the EPS of Maraston (2005). The SSPs used in this work cover 12 ages, $t=0.01,0.03,0.05,0.1$, $0.2,0.5,0.7,1,2,5,9,13 \mathrm{Gyr}$, and 4 metallicities, namely: $Z=0.02 Z_{\odot}, 0.5 Z_{\odot}, 1 Z_{\odot}$ and $2 Z_{\odot}$, summing up 48 SSPs.

When trying to describe the continuum observed in AGNs, the signature of the central engine cannot be ignored. Usually, this component is represented by a featureless continuum ( $F C$, e.g.Cid Fernandes et al. 2004 and references therein) of power-law form that follows the expression $F_{\nu} \propto \nu^{-1.5}$. Therefore, this component was also added to the base of elements. In the spectral region studied here, hot dust plays an important role in the continuum emission of active galaxies. Previous studies (i.e., Riffel et al. 2006, for instance) report a minimum in the continuum emission around $1.2 \mu \mathrm{m}$, probably associated with the red end of the optical continuum related to the central engine and the onset of the emission due to reprocessed nuclear radiation by dust (Riffel et al. 2009 and references therein). In order to properly account for this component, we have included in our spectral base 8 Planck distributions (black-body- $B B$ ), with $T$ ranging from 700 to $1400 \mathrm{~K}$, in steps of $100 \mathrm{~K}$.

\section{Results}

In this work we investigate the NIR spectra of 24 Seyfert galaxies (9 Sy 1 and 15 Sy 2) observed with the IRTF SpeX, obtained in the short cross-dispersed mode. The results of the spectral synthesis fitting procedure are presented and discussed in details in Riffel et al. (2009). The approach followed here is based on the STARLIGHT code (Cid Fernandes et al. 2004,2005, and references therein), which considers the whole observed spectrum, continuum and absorption features.

In genneral, the spectral synthesis shows that the NIR continuum of active galaxies can be explained in terms of at least three components: a non-thermal continuum, the dust emission and the stellar population of the circumnuclear region. As can be seen in Fig. 1, the contribution of the latter to the nuclear continuum is higher than $50 \%$ in most objects. Therefore, its study is a critical step in the analysis of the continuum emission of Seyfert galaxies. Moreover, our results are consistent with the predictions of the unified 

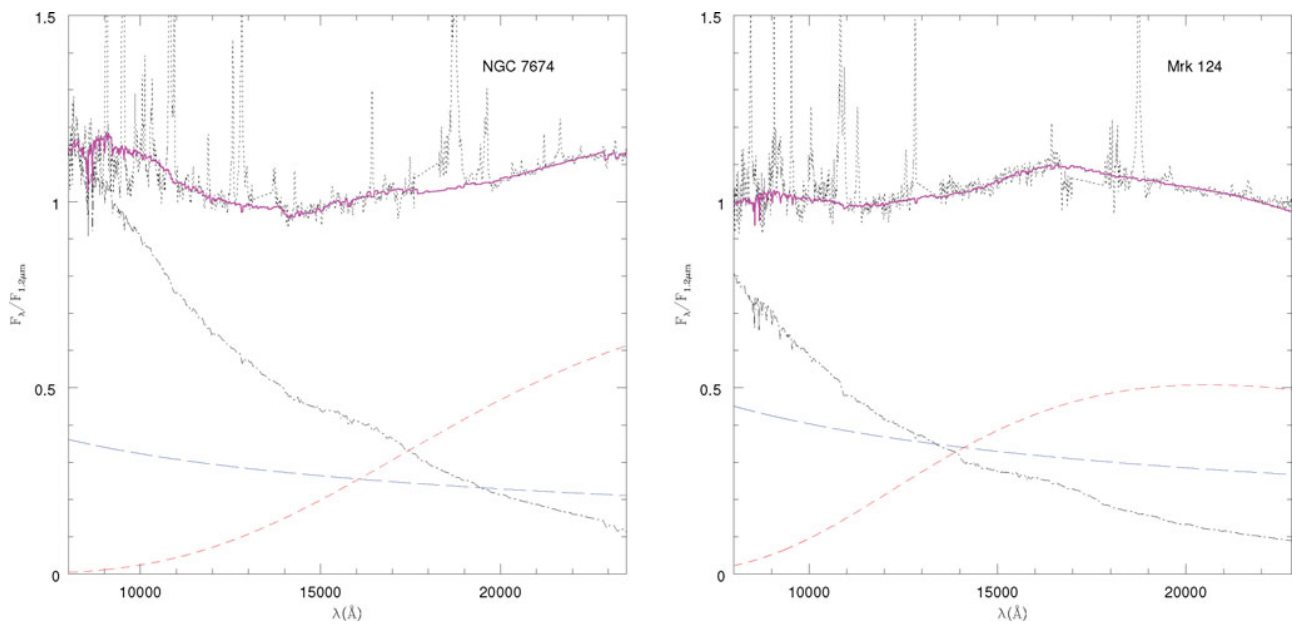

Figure 1. Three continuum components of NGC 7674 and Mrk 124. Dot-short dashed line represents the stellar population $\left(x_{\mathrm{y}}+x_{\mathrm{I}}+x_{\mathrm{o}}\right)$. The $\mathrm{FC}$ and hot dust component are represented by the long and short dashed lines, respectively. The solid line is the sum of the three components and the dotted line represents the observed spectrum.

model for AGNs, as the non-thermal continuum and the hot dust emission are present in all Sy 1 sources and only in a small fraction of the Sy $2 \mathrm{~s}$.

Note that to take into account noise effects that dump small differences between similar spectral components, we present our results using a condensed population vector, which is obtained by binning the $\vec{x}$ into young, $x_{Y}\left(t_{j} \leqslant 5 \times 10^{7} \mathrm{yr}\right)$; intermediate-age, $x_{I}$ $\left(1 \times 10^{8} \leqslant t_{j} \leqslant 2 \times 10^{9} \mathrm{yr}\right)$ and old, $x_{O}\left(t_{j}>2 \times 10^{9} \mathrm{yr}\right)$ components, using the flux contributions. We have also binned the black-body contributions into two components. The cool $\left(B B_{c}\right)$ is obtained by summing up the $B B$ contributions with $\mathrm{T} \leqslant 1000 \mathrm{~K}$, and the hot one $\left(B B_{h}\right)$ with $\mathrm{T} \geqslant 1100 \mathrm{~K}$. For more details on vector definition see Riffel et al. (2009).

Regarding the stellar population component our results point to a mean metallicity solar to above solar, if we consider the light-weighted values, while for the mass-weighted mean metallicity our results indicate a sub-solar value. We associate this discrepancy with the well known age-metallicity degeneracy, i.e. for a fixed mass, a high-metallicity stellar population looks cooler - and older - than a low-metallicity SP, thus resulting in a higher $M / L$ ratio. Moreover, this is consistent with a galaxy chemical enrichment scenario in which the young population is enriched by the evolution of the early massive stars. In this context, the light-weighted metallicity is more sensitive to the young component, while the mass-weighted metallicity to the old stellar population.

\section{Conclusions}

The main results can be summarised as follows:

- We found evidence of correlation among the $\mathrm{W}_{\lambda}$ of SiI $1.59 \mu \mathrm{m} \times \mathrm{Mg} \mathrm{I} 1.58 \mu \mathrm{m}$, equally for both kinds of activity. Part of the $W_{\mathrm{NaI} 2.21 \mu \mathrm{m}}$ and $W_{\mathrm{CO} 2.3 \mu \mathrm{m}}$ strengths and the correlation between $W_{\mathrm{NaI} 2.21 \mu \mathrm{m}}$ and $W_{\mathrm{MgI} 1.58 \mu \mathrm{m}}$ appears to be accounted for by galaxy inclination.

- For the 7 objects in common with previous optical studies (based on the same method of analyses), the NIR stellar population synthesis does not reproduce well the optical results (see Fig. 2). 


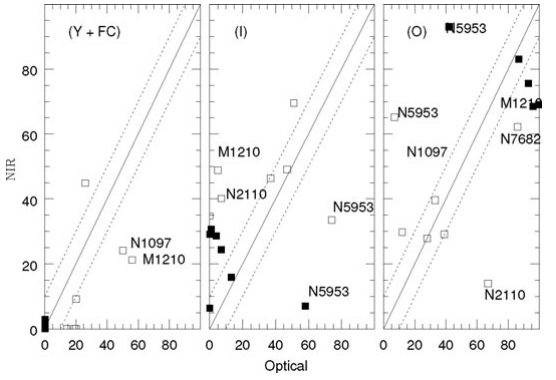

Figure 2. Comparison of the population vectors obtained in the NIR (this work) and in the optical (CF04), for 7 objects in common. The symbols indicate the population vectors. The full line is the identity line, the dotted lines represent $\pm 10 \%$ deviation from the identity. Open and filled symbols are the flux and mass fraction, respectively.

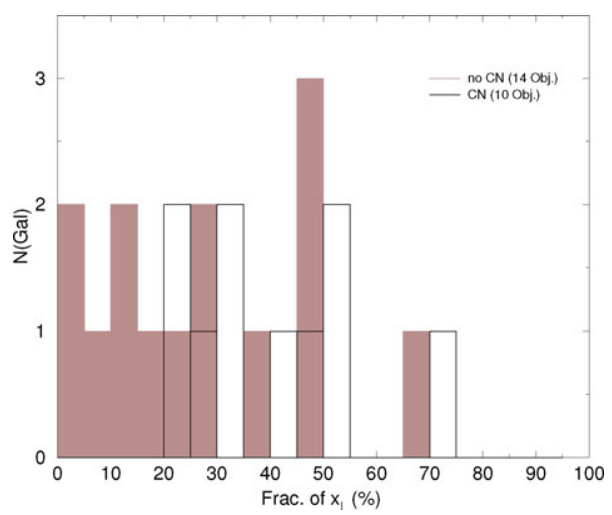

Figure 3. Histograms comparing the intermediate age component of the galaxies with $\mathrm{CN}$ detection (empty histogram) and un-detection (shaded).

- Our synthesis shows significant differences between Sy 1 and Sy 2 galaxies. The hot dust component is required to fit the $K$-band spectra of $\sim 80 \%$ of the Sy 1 galaxies, and only of $\sim 40 \%$ of the Sy 2 . Besides, about $50 \%$ of the Sy 2 galaxies require a featureless component contribution in excess of $20 \%$, while this fraction increases to about $60 \%$ in the Sy 1 . Also, in about $50 \%$ of the Sy 2, the combined FC and $X_{Y}$ components contribute with more than 20\%, while this occurs in $90 \%$ of the Sy 1 . This suggests recent star formation (Cid Fernandes et al. 2005) in the central region of our galaxy sample.

- We found that the light at $1.223 \mu \mathrm{m}$ in central regions of the galaxies studied here contain a substantial fraction of intermediate-age SPs with a mean metallicity near solar. Moreover, our analysis confirms that the $1.1 \mu \mathrm{m} \mathrm{CN}$ band can be taken as an unambiguous tracer of intermediate-age stellar populations.

What emerges from this work is that the NIR may be taken as an excellent window to study the stellar population of Sy 1 galaxies, as opposed to the usually heavily attenuated optical range. Our approach opens a new way to investigate and quantify the contribution of the three most important NIR continuum components observed in AGNs. This new thecnique is described in Riffel et al. (2009).

\section{References}

Cid Fernandes, R., Mateus, A., Sodré, L. et al. 2005, MNRAS, 358, 363

Cid Fernandes, R., Gu, Q., Melnick, J., Terlevich, E. et al. 2004, MNRAS, 355, 273

Iben, I. \& Renzini, A. 1983, ARA\&A, 21, 271.

Lançon, A. \& Wood, P. R. 2000, A\&SAS, 146, 217.

Maraston, C. 2005, MNRAS, 362, 799.

Origlia, L., Moorwood, A. F. M., \& Oliva, E. 1993, $A \mathscr{G} A, 280,536$.

Origlia, L. \& Oliva, E. 2000, NewAR, 44, 257

Riffel, R., Rodríguez-Ardila, A., \& Pastoriza, M. G. 2006, A\&\&A 457, 61.

Riffel, R., Pastoriza, M. G., Rodríguez-Ardila, A., \& Maraston, C. 2007, ApJ, 659L, 103

Riffel, R., Pastoriza, M. G., Rodríguez-Ardila, A., \& Maraston, C. 2008, MNRAS, 388, 803.

Riffel, R., Pastoriza, M. G., Rodríguez-Ardila, A., \& Bonatto, C. 2009, arXiv:090\%.4144.

Schmidt, A. A., Copetti, M. V. F., Alloin, D., \& Jablonka, P. 1991, MNRAS, 249, 766. 